\title{
Vișualización de información y cadena de significados. Cartografía de Infovis orientada a su experimentacion didáctica en procesos de diseño
}

Information visualization and chain of meanings. Infovis cartography oriented

to its didactic experimentation in design processes

\author{
> Ms. Arq. Andrea De Monte \\ Profesora / Investigadora \\ Facultad de Arquitectura, Diseño y Urbanismo. U.N.L. Argentina \\ andreademonte@sdarquitectos.com.ar
}

\begin{abstract}
The digitization of culture imposes new logic to the design disciplines in professional and academic areas. New roles, competencies and requirements emerge in front of an architecture that today is essentially material and also informational. The overabundance of information confuses and forced to develop strategies and skills that go beyond the traditional system of reading and observation to achieve its analysis and understanding. In this sense, the work investigates and cartography variables of information visualization related to semantic attributes, aimed to recognize their potential for instrumental experimentation in design processes within learning environments.
\end{abstract}

Keywords: Base De Datos; Visualización; Semántica; Aprendizaje

\section{Introducción}

El presente trabajo es parte de la investigación realizada en el contexto de la Tesis de Maestría (De Monte 2013), el cual actualmente se continúa en líneas de investigación más amplias en el ámbito de la FADU. El objetivo principal es indagar estrategias pertinentes para la adquisición de hábitos y prácticas que incorporen instrumental y creativamente recursos de los medios digitales en la práctica y aprendizaje disciplinar. La hipótesis planteada en la Tesis enuncia que recursos de Visualización de información (Infovis) podrían aplicarse, en didácticas innovadoras, como instrumentos que potencien el aprendizaje en procesos de diseño. La misma se sostiene en la necesidad de desarrollar incumbencias y destrezas para dotar de sentido la gran cantidad de información disponible a partir de la proliferación de bases de datos (BD) de todo tipo. Se considera necesario en poblaciones estudiantiles "nativas digitales", aprender a discernir, establecer criterios y jerarquías para lograr interpretaciones que colaboren en los procesos de aprendizaje a partir de la experimentación creativa del poder que nos ofrecen los medios digitales para expandir nuestros sentidos y capacidades cognitivas.

La cartografía propuesta organiza Infovis relacionando sus aspectos esenciales y variables semánticas vinculadas. El sentido fue orientar su articulación en didácticas que las exploren con el objetivo de validar su posibilidad instrumental en procesos de diseño. El trabajo se complementó con experiencias prácticas en talleres de grado, en carreras de diseño y arquitectura, donde las mismas se pusieron a prueba en intersticios de los procesos de aprendizaje.
Infovis y cadenas de significado

En contextos complejos, multideterminados y con superabundancia de información Infovis se presenta como un dispositivo o medio que colabora en el proceso para su comprensión. La información conformada en $\mathrm{BD}$ a partir de operaciones prácticas y conceptuales se traduce en representaciones visuales con el objetivo de comunicar y hacerlas inteligibles evidenciando patrones, tendencias y anomalías, constantes o variables. La Infovis es una disciplina emergente y versátil en la que confluyen diversos campos relacionados con el diseño visual, la comunicación, la programación, el arte, etc. Ha logrado gran difusión en los últimos 15 años en el contexto de una cultura hipervisual y se ha visto favorecida por la accesibilidad y evolución del hardware, el desarrollo del software y la necesidad de hacer inteligible y útiles la gran cantidad de BD disponibles a través de las redes en tiempo real. Este trabajo considera que el objetivo de Infovis, es colaborar a la comprensión de estas formas simbólicas de la cultura contemporánea (Manovich 2006) para lograr transformar la información en conocimiento, siendo el atributo clave que la diferencia de otros medios, la posibilidad que brinda de evidenciar aspectos, patrones o relaciones no perceptibles por otras formas de representación. Infovis opera como micro unidades de comprensión, las que puestas en un contexto determinado, configuran cadenas de significados. Al ser entendidas como unidades narrativas permiten avanzar en una trama determinada hacia la construcción de cierto conocimiento, paradójicamente, dentro de la lógica anti narrativa propia de la Web (Manovich 2006). Desde este lugar es pertinente proponer Infovis, 


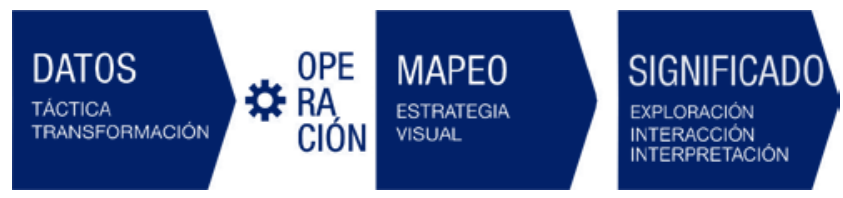

Figura 1: Infovis y Cadena de significados.

como cadena de significados, articulada en didácticas orientadas al ámbito del aprendizaje disciplinar. Considerando que el diseño requiere, por su especificidad, contemplar múltiples variables y escalas que confluyen superponiéndose en una práctica que le es propia, Infovis se presenta potencialmente como interface apta para colaborar en los procesos. En este sentido es válido reconocer que toda estructura de pensamiento graficable, en tanto sistema de relaciones de organización, siendo grafo, signo, símbolo, o señal impulsa formas emergentes de pensamiento. En esta capacidad de reconfigurar el lenguaje permite introducir variaciones nuevas, desarrollar múltiples alternativas, así como construir metodologías complejas. (Cadalso 2004:364)

A partir de antecedentes relevados en la práctica profesional de ciertos estudios (OMA, UnStudio, MRDV, FOA, etc.) se argumenta la incidencia que Infovis adquiere, no solo en aspectos de la comunicación, sino también como diagramas de notación y catalizador de acciones proyectivas. Así mismo colabora en la definición de premisas de diseño objetivas y certeras, optimizando las estrategias conceptuales de diseño y planificación a partir de la visión intencionada. La interpretación, detección de modelos, métodos y procedimientos devenidos del análisis de relevamientos diversos, Ej.: de experiencias y flujos de movimiento en el espacio urbano o edilicio, de accesibilidad, distribución de objetos o sitios atractores, de usos de la tierra, ubicación de nodos de transporte etc. (ej. http://www.spacesyntax.com/our-approach/ v. 09-09-2014). Infovis integra $\mathrm{BD}$ de distinta naturaleza y facilita su edición y superposición en layers ofreciendo visiones de apertura al análisis de situaciones complejas, multideterminadas, y totalizadoras. El registro de patrones no visibles o intangibles son perceptibles, mensurables y por lo tanto susceptibles de ser manipulados. Distintas variables pueden incidir en el proyecto, por ejemplo sobre la percepción fenomenológica o la apropiación de los espacios. Es viable contemplar y evaluar la incidencia de las sombras, como huellas del sol, los vientos, la ergonomía etc. entre otras variables ambientales o antropométricas. Condiciones que traducen la mecánica solar en relaciones trigonométricas proyectuales, transformando la sombra en elemento material manipulable del diseńo. (http://pvgarquitectos. com/prehome.php 09-09-2014). Esta apretada síntesis de aspectos enumerados se consideran fundamentales para ser incorporados en el aprendizaje disciplinar. Variables que, vinculados a Infovis y sostenidos en la destreza natural (no orientada) que poseen nuestros alumnos nativos digitales, permiten vislumbrar un área fecunda para explorar en didácticas que se integren en los talleres de diseño.

\section{Experimentación didáctica y mapa de referencia}

Infovis representa un campo de diseño en sí mismo en tanto interface de acceso a datos abstractos y conceptuales. Así mismo por sus características se presenta como un recurso orientado a la investigación aun en desarrollo y oportunidad para su incorporación instrumental tanto en los procesos de aprendizaje, como efectivamente en la práctica disciplinar. Su versatilidad y adaptación a distintas temáticas (áreas de la estadística y científicas, de la comunicación, periodismo, arte, etc.) pone en debate su incumbencia, funciones y objetivos, así como cuáles son las cualidades que deben comprender o los objetivos que las definen. Gran variedad de rangos, distancias conceptuales y epistemológicas rodean el tema, ofreciendo matices y variantes para catalogar su universo. Algunos lo hacen a través de valoraciones empíricas relacionadas a las características percibidas como similares (Lhose; Biolsi, 1994) otros basan su análisis considerando los formatos gráficos, los contenidos o el contexto de aplicación. ( "La Tabla periódica de métodos de visualización” de Lengler y Eppler 2007). Desde la infoestetica las estudian en relación a las características de la representación, los valores estéticos y la interacción (Vande Moere 2007). Finalmente los repositorios ordenan por Temas, Método o Forma (sintaxis de diseño), Autores, Años o Herramientas. Los ejemplos muestran la variedad de rangos, distancias conceptuales y epistemológicas que existen sobre el tema, evidenciando en la transversalidad temática, su mayor riqueza productiva, pero también su mayor limitante epistemológico (Córdoba C. 2012). Estudios en este sentido se están proponiendo desde ámbitos académicos ante la necesidad de fundar una teoría, taxonomía y métodos para su evaluación que la sustenten y la proyecten a futuro como una disciplina. Frente a este campo sumamente diverso fue necesario elaborar una Cartografía o mapa de referencia que incorpora al análisis variables semánticas con el sentido de experimentar su puesta en acción en ámbitos de formación. Pensar la Infovis como instrumento para el aprendizaje disciplinar, implicó reconocer propiedades, características esenciales y formas de operar con el fin de diseñar estrategias prácticas y detectar contextos de aplicación.

\section{Hacia una Cartografia de Infovis orientada}

Esta propuesta analiza y ordena Infovis a partir de su estudio para este caso específico, fundamentada en una selección de antecedentes considerados relevantes en relación a los objetivos pedagógicos del trabajo. (Manovich, 2010; Vande M., 2007,2012; Lengler,2007; Lima,2010) Se complementó con el análisis más exhaustivo de 40 casos específicos de Infovis sobre una selección de ejemplos de referencia, que permitieron verificar las categorías enunciadas.

Existen dos principios que se han mantenido constantes en la práctica Infovis desde sus inicios, en la segunda parte del siglo XVIII y son esenciales en su definición, Aunque probablemente no contengan todas las formas posibles actualmente de Infovis, resultan suficientes para definir características que le son propias frente a otras técnicas y tecnologías de representación visual. Estas son: las operaciones de reducción que se aplican sobre los atributos de las BD que se representan y las características del espacio de representación en donde se privilegia la dimensión espacial (posición, tamaño, forma, curvatura, movimiento), con una clara preferencia de la geometría y topología, por sobre otras dimensiones visuales (colores, tonos, transparencias etc.), para representar las diferencias 


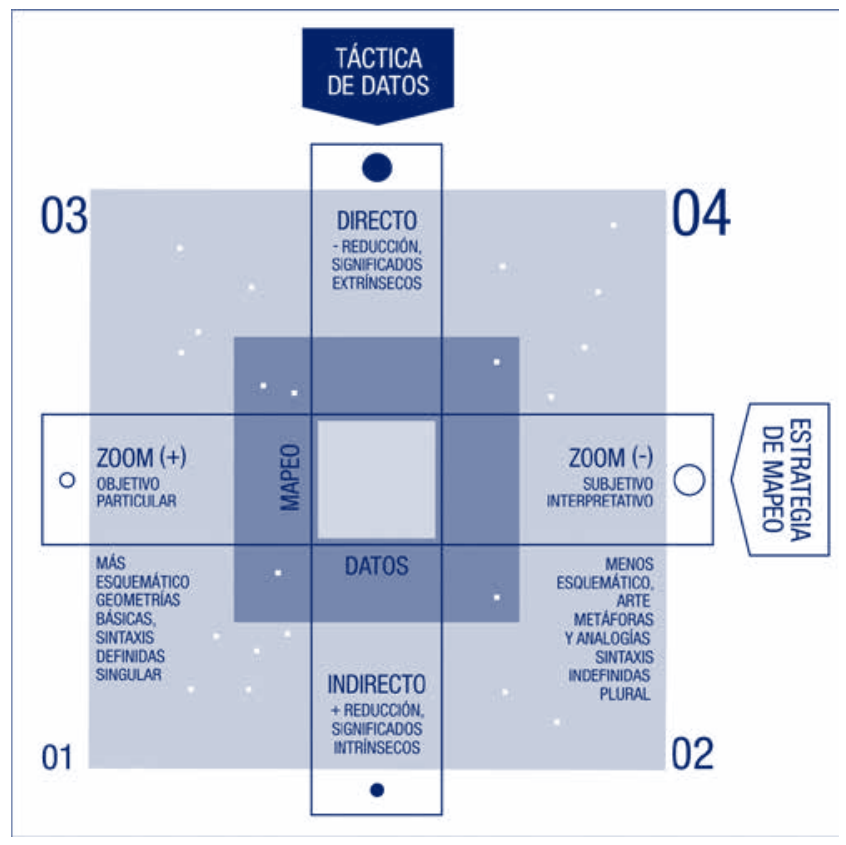

Figura 2: Esquema espacializado de la Cartografía propuesta.

claves y revelar los patrones o relaciones más significativas (Manovich 2010). Es posible establecer entre estos principios formas relacionadas con variables semánticas que devienen en modalidades de comprensión o tipos de interpretación susceptibles de ser decodificadas. La cartografía se organiza a partir de reconocer que Infovis opera esencialmente entre capas interrelacionadas, que dependerán de la intención del diseñador y del contexto dado por los fines u objetivos de la misma. Estas capas se definen como A. Táctica de los datos y B. Estrategia del mapa vinculados a partir de C. Operaciones e instrumentos gráficos utilizados.

\section{A. Táctica de los datos}

Establece los criterios generales en la forma y particularidades en que se incorporan y manipulan las $\mathrm{BD}$. La variable se polariza entre formas con tácticas que van del tipo directo hasta el indirecto Manovich (2010). Ambas, a su vez, se relacionan con la capacidad de facilitar en los usuarios lecturas de significados intrínseco o extrínseco a los mismos.

Táctica de datos indirecta: Son aquellas que operan reducción en las cualidades de las BD a representar. En su operación de transformación requieren criterios de filtrado, selección y cuantificación para definir la cualidad a representar. Los datos se presentan sustituidos por figuras geométricas simples (gráficos vectoriales). La forma de su representación es esquemática y abstracta. Poseen menor riqueza en cuanto a las cualidades del dato representado y como contrapartida, adquieren claridad y son eficaces en la comunicación del aspecto o cualidad del dato seleccionado. Los significados que se comunican responden específicamente a un objetivo y de esta manera permiten al usuario comprender aspectos intrínsecos del mismo (Van de Moere, 2007). La táctica que opera sobre significados intrínsecos habilita lecturas enfocadas, su objetivo es facilitar la rápida comprensión disponiendo aspectos claves sintetizados de los mismos. Facilita la comparación y contraste de información cuantificada, así como la superposición de distintos aspectos o cualidades para comprender una situación o las aristas de un problema complejo a partir del análisis de partes.

Táctica de datos directa: Son aquellas que no operan reducción para ser representados sino que las $\mathrm{BD}$ son incorporadas con todas (o varias simultáneamente) de sus cualidades originales. La reducción que se aplica es de tipo cuantitativo, esto es, con relación a las cantidades posibles de ser procesadas, o a la pauta del diseńador para el muestreo. Los datos al conservar sus cualidades ya no admiten ser traducidos en un sentido único o forma determinada, y conservan la mayoría de las veces su forma (representación visual) original. Pueden también, en ciertos casos adquirir variantes en este sentido distanciándose de las mismas hacia formas totalmente aleatorias vinculadas a la sensibilidad del diseñador. Las posibilidades de interpretación son más libres o abiertas y se definen promoviendo significados extrínsecos al dato visualizado. Los significados extrínsecos son factibles a partir de incluir todas las cualidades de los datos y permiten detectar patrones que subyacen o son menos evidentes en los mismos invocando la reflexión para su comprensión. Generalmente, se relacionan con principios artísticos en su representación y ofrecen la posibilidad de lecturas y significados que van por fuera o que son más amplios que los datos en sí mismos. La forma de su representación al expresar datos cualificados es diversa y variable, sujeta al diseño visual o de programación de algoritmos. Para su operatividad requieren métodos de procesamiento (software) y usualmente un mayor equipamiento tecnológico (hardware).

\section{B. Estrategia del mapa}

Refiere a la forma y uso del espacio de representación que define la lógica resultante en la aplicación del mapeo de datos. Implica variables en la representación que van, como un mecanismo de aumento en la escala del enfoque: zoom (+), hacia una reducción de signo opuesto: zoom (-). Ambos polos se relacionan con la posibilidad de comprender aspectos de forma particular-objetiva (singular) o interpretativa-subjetiva (plural).

Estrategia del mapa: zoom(+): Propone una visión de mayor acercamiento y enfoque. Presenta un recorte de cualidades específicas, dentro de un rango amplio de las $\mathrm{BD}$, y tiende a una forma de comprensión particular y objetiva de sus aspectos. Para los datos ingresados con reducción y cuantificados (visualización indirecta), las variables de mapeo serán de forma definida principalmente por atributos topológicos y geométricos. Se privilegia el uso de dimensiones espaciales, como distancia entre elementos, posición, tamańo, curvas para aspectos claves, y el uso de otras dimensiones visuales, como tonos, colores, transparencias, rellenos, etc., para propiedades menos relevantes. La comprensión es en general singular y directa enfocada a ciertas cualidades de los datos. Su mayor valor está dado en un poder de comunicación eficiente ordenando por rangos o jerarquías para visualizar, comparar y contrastar información, como forma de interpretación y análisis facilitando la superposición de capas de información de distinta índole, entre otras cosas. Se relacionan directamente con la táctica de los datos ingresados con reducción en forma indirecta. 
Estrategia del mapa: zoom (-): Indica una visión de mayor distancia, y por ello menor acercamiento, sobre mayores extensiones de datos. Su enfoque es amplio ofreciendo visiones que tienden a lo general. La comprensión es a partir de una interpretación generalmente subjetiva del usuario.

Los datos representados preservan la forma y muchas de sus cualidades de origen. Por lo que pueden ser manipulados por parámetros específicos definidos por el usuario. Las características en las formas y uso del espacio en los mapeos son la variabilidad y diversidad en su diseño. Variaciones en sus atributos de dimensión espacial, visual y temporales se conservan solo en ciertos casos de narrativas musicales o textos, por ejemplo. Tienden a ser menos esquemáticos y remitirse a recursos como las metáforas, analogías, etc. para su representación. El despliegue y la forma de presentar los datos favorece inferir variables de comprensión múltiples e interpretaciones subjetivas, vinculadas a los conocimientos previos del usuario. Su mayor valor está dado por sus cualidades estéticas e interpretativas, así como las relaciones, vínculos y patrones que infieren. Aspectos relacionados a la exploración, en tanto posibilidades de variación y control de parámetros utilizando datos externos (simulaciones matemáticas, diseño de algoritmos, sonidos, etc.) son asimismo particularidades que las definen. Se relacionan con la táctica de los datos ingresados sin reducción denominada directa.

\section{Las operaciones. Instrumentos gráficos de visualización}

Las operaciones a realizar para visualizar información comprenden los instrumentos para traducir y mapear datos. Algunas operan hacia visualizaciones de tipo estático, como gráficos bimedia, diagramas, infografías o mapas conceptuales y pueden resultar de recursos análogos o digitales. En otros casos son interactivas y personalizables, o permiten combinar varios tipos de visualizaciones en un mismo layout. Pueden igualmente resultar del diseño de algoritmos a veces disponibles en plataformas en línea para usuarios no expertos o responder a diseños de programación complejos y sofisticados. Vinculado al análisis realizado para cartografiar la Infovis, se podrían en un estudio más extenso establecer algunas líneas generales y coincidentes entre el tipo de instrumento que se utiliza, las tácticas de los datos implementadas y las estrategias del mapa propuesto.

La Figura 2 espacializa las interrelaciones enunciadas entre la Táctica de los datos directalindirecta y los significados que se infieren de los mismos extrinsecos/intrínsecos, en relación con la Estrategia del mapa resultante en visiones zoom (-) / zoom (+) y la forma de comprensión en la comunicación que promueven objetiva-particular / subjetiva-interpretativa. Tanto el tipo como la forma (cualidades) de los datos definen la estrategia del mapeo, que significan a su vez una estrategia para la comunicación y comprensión de los mismos, como puede suceder a la inversa. La promoción de modalidades de comprensión significativa evidencia una tensión predominante entre los cuadrantes 01 y 04 conformando los extremos de mayor contraste. Fig. 3.

Área 01: Caracterizada por formas directas y objetivas de las partes pertenecientes a un todo más amplio a partir de aplicar un criterio de selección y cuantificación sobre cualidades específicas, facilitando y ampliando de este modo su análisis, comparación e

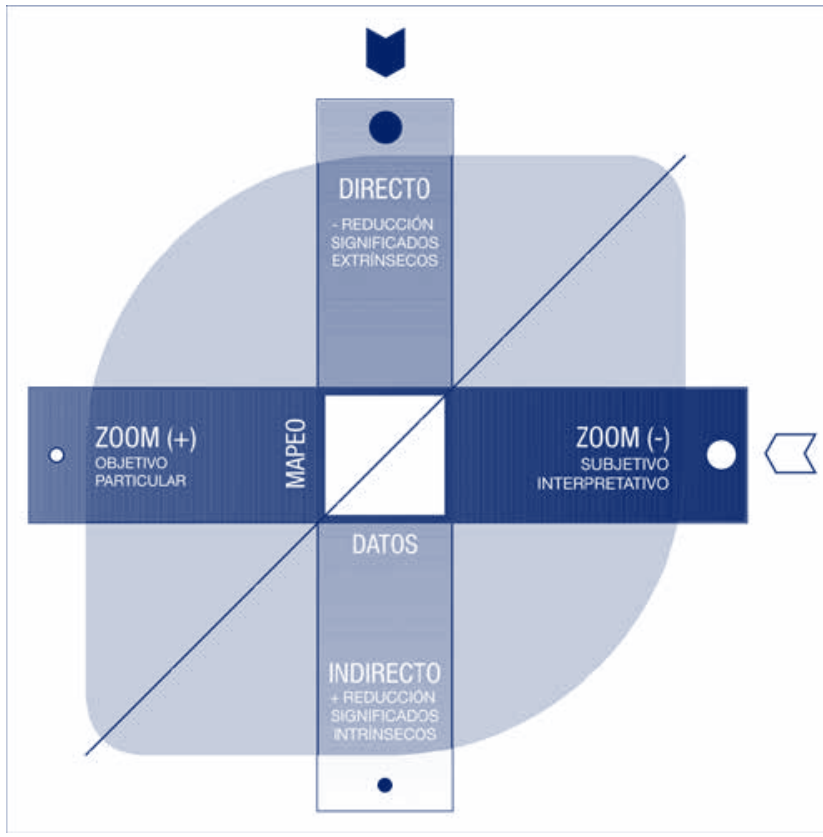

Figura 3: Síntesis de las tendencias opuestas y principales

interpretación Construyen cadenas de significados de tipo objetivo e intrínseco sobre los datos. Área 04; Caracterizada por formas indirectas y subjetivas, a partir de la interpretación personal e intuitiva de los usuarios o de las intenciones en el despliegue y diseño. La manipulación de cualidades y el criterio de interpelación construyen cadenas de significados de tipo interpretativo y extrínseco. Entre ambos extremos, áreas 02 y 03, se presenta una extensa zona que contiene alternativas con diferentes niveles de influencias de las variables analizadas, según su proximidad a los polos de los ejes establecidos.

\section{Aportes y conclusiones}

El enfoque del trabajo consolida a Infovis como posibilidad de acción en didácticas estratégicas. A partir de construir metáforas visuales permitiría ver cómo conjuntos de datos, ideas y relaciones, traducidos en elementos visuales comprensibles, configuran cadenas de significado y habilitan interpretaciones multi-escala, con variedad de matices o imprevisibles por otros medios. Confiere relevancia, sintetizando y facilita la toma de decisiones con criterios objetivos y rapidez, en visiones multi-capa de informaciones solapadas en procesos de diseño. Puesta en contexto como instrumento en los procesos de aprendizaje (Stipech 2010) (proyectuales, para la comunicación, diseño, investigación, etc.) promovería instancias de interpretación y comprensión configurándose como un medio valido de representación (lenguaje) para el conocimiento. La Cartografía aporta una visión que establece vínculos entre principios esenciales de Infovis y cualidades semánticas, colaborando a su inclusión estratégica en prácticas pedagógicas. Con este sentido se distinguen modalidades en Infovis vinculadas a variaciones en las características de las cadenas de significado resultantes de la interacción entre las tácticas de los datos y las estrategias de 
mapeo. Es posible inferir su posibilidad de instrumento versátil, innovador y pertinente articulado en procesos de aprendizaje disciplinar. En este sentido ofrece un campo de potencialidades válidas a considerar como:

1. Capacidad de develar patrones que subyacen a los datos y a sus relaciones (Promoción de Inteligencia relacional y reconocimiento de vínculos explícitos o subyacentes).

2. Extracción de inferencias de la forma visual para construir conocimiento. Interpretación intuitiva como anclaje para nuevos conocimientos.

3. Interpretación de relaciones entre conceptos y significados en red para construir unidades de sentido.

4. Abstracción y síntesis para la comprensión significativa de información clasificada o compleja y superpuesta.

5. Promoción del desarrollo de criterios y sentido para lograr interpretar y comprender.

6. Visibiliza sintéticamente datos claves facilitando, con economía de tiempo y predictibilidad, diagnósticos y acciones proyectivas.

7. Promoción de una reflexión que induce a conceptualizar a partir de pensar haciendo. (Procesar y diseñar de Infovis).

8. Construcción de conocimiento mediante operaciones de síntesis, comparación, superposición y cambios de escala.

9. Construcción colectiva de conocimiento, reinterpretación y mixtura de recursos (información) configurando un lenguaje visual, con atributos de interacción, susceptible de compartirse y comunicarse a través de las redes.

\section{Referencias}

Cadalso, E.; Haiek Coll, A. y Soza Ruiz, P. (2004). Graficando estructuras de conocimiento: Diagramas matriciales, infomapas, cartografías y estructuras de organización cognitiva. VIII Congreso SIGRADI. Brasil. Ed. Unisinos. pp. 363-366.

Card, S.K.; Mackinlay Jock, D. y Shneiderman, B. (1999). Readings in Information Visualization: Using Vision to Think. EE. UU. Ed. Morgan K.
Córdoba Cely, Carlos; Alatriste M., Yadira (2012). Hacia una taxonomía de investigación entre Visualización de Información y Diseño. En: No Solo Usabilidad, no 11, 2012. http:// nosolousabilidad.com

De Monte Andrea (2013). Intersticios en el aprendizaje de la arquitectura. La visualización de datos como instrumento en el proceso proyectual. Tesis de Maestría. MDPI, Facultad de Arquitectura UCC. En proceso de publicación.

García, M. (ed.) (2010). The Diagram of Arquchitecture. Londres: Reader.

Lengler, R. y Eppler, M.J. (2007). Towards a periodic Table of visualization Methods of Management. Proccedeedings of Graphics and Visualization in Engineering. Clearwaer. Florida. Ed. ACTA Press.

Lima, M. (2011). Information Visualization Framework. Visual Complexity,VCBlog(v.09-09-2014)En: http://www.visualcomplexity.com/vc/blog/?p=1076

Manovich, L. (2006). El lenguaje de los nuevos medios de comunicación. Buenos Aires. Ed. Paidós Communication.

Manovich, L. (2010). What is Visualization? Poetess Archive Journal 2.RetrievedJuly4,2012.En: http://manovich.net/2010/10/25/ new-article-what-is-visualization/ (v. 13-04-2014).

Piscitelli, A.; Adaime, I.y Binder, I. (2009). El Proyecto Facebook y la postuniversidad. Sistemas operativos sociales y entornos abiertos de aprendizaje. Colección Fundación Telefónica. Buenos Aires. Ed. Ariel.

Stipech, A. (2012). Fundamentos de Cátedra Introducción a los Medios Digitales. FADU-UNL. Santa Fe. En: http://www. fadu.unl.edu.ar/imd (v.10-09-2014).

Vande Moere A., Tomitsch M., Wimmer C., Boesch C. and Grechenig T. (2012), "Evaluating the Effect of Style in Information Visualization", Proceedings IEEE Infovis. Transactions on Visualization and Computer Graphics, pp.2739-2748.

Vande Moere, A. y Lau, A. (2007). “Towards a Model of Information Aesthetic Visualization”. IEEE International Conference on Information Visualisation (IV'07). Zurich. Suiza, pp. 87-92. 